\title{
The feasibility of an early hospital discharge following chemotherapy for the acute myeloid leukemia
}

\author{
Sopko L, Al Sabty F, Rimajova V, Roziakova L, Elanova A, Demeckova E, Mistrik M \\ Department of Hematology and Transfusion Medicine, Faculty of Medicine, University Hospital, Comenius \\ University, Bratislava, Slovakia. sopko.ladislav@gmail.com
}

\begin{abstract}
Objective: The hospitalization of the patient during the critical myelosuppressive period after chemotherapy is often complicated by infections caused by nosocomial pathogens, what is associated with a high antibiotics consumption and with prolongation of the period of hospitalization. These findings have led many centres to change their policy from "in-hospital" to "out-hospital care". In this retrospective study we tried, on the basis of our experiences, to identify the feasibility and safety of this approach.

Patients and methods: We studied 56 patients with the acute myeloid leukemia treated in our clinic with the consolidation chemotherapy. We compared two groups of patients. In the first group, the patients were discharged upon completion of chemotherapy, consequently followed up as outpatients. Patients in the second group were observed in hospital during the entire nadir. Following 41 courses, patients were discharged and instructed to return immediately if fever or any other change of their clinical status occurred.

Results: In 24 cases after chemotherapy, the patients returned to the hospital after a discharge (in 23 cases because of fever), in 17 cases of nadir periods the hospitalisation was not necessary at all. Seven patients were readmitted in septic shock, but rapidly recovered. Two other patients died, one due to an irreversible shock within 12 hours of readmission and one due to bacterial meningitis within 48 hours after readmission. In 10 cases of rehospitalization, patients responded to the first line of antibiotics. In the second group of the patients, only 2 courses of consolidation from a total of 15 were not complicated. In contrast to the first group, we detected only poor effectiveness of broad-spectrum antibiotics in the group of inpatients.

Conclusions: For AML patients in a good clinical status without any complicating medical conditions, the early discharge is feasible, safe and cost saving option (Tab. 2, Fig. 2, Ref. 7). Full Text in PDF www.elis.sk.

Key words: early discharge, febrile netropenia, acute myeloid leukemia, consolidation chemotherapy.
\end{abstract}

The treatment of the acute myeloid leukemia is a very difficult and long-term issue. It consists of repeated administration of myelosupresive chemotherapy. In the leukopenic period, the patients are at a high risk of life-threatening infections or bleeding. When we see an increasing incidence of infections caused by multiresistant nosocomial bacteria, it is appropriate to ask how to protect patients against these dangerous pathogens. Several reports have questioned the necessity of keeping patients in hospital after chemotherapy until a full neutrophile count recovery. One option, how to decrease the risk of these infections, is an early discharge of patients from hospital. In this retrospective study, we present our experiences with this method. We attempted to find the answer about safety and feasibility of this policy and also to identify potential risks of such approach.

\section{Patients and methods}

Patients were treated in ordinary wards during the induction phase until the achievement of a hematologic remission. The chemotherapy usually consisted of anthracycline and cytarabine,

Department of Hematology and Transfusion Medicine, Faculty of Medicine, University Hospital, Comenius University, Bratislava, Slovakia

Address for correspondence: L. Sopko, MD, Department of Hematology and Transfusion Medicine, Faculty of Medicine, University Hospital, Comenius University, Antolska 11, SK-851 07 Bratislava, Slovakia. followed by either high-dose cytarabine based consolidation chemotherapy or stem cell transplantation, if indicated. When the consolidation chemotherapy was completed, patients were offered two opportunities: to go home or to stay in hospital. For all patients, who received the offer to go home, we followed these criteria: - no signs of infections or bleeding, - good physical conditions, - no intravenous therapy.

If the subject chose to leave the department and went home, we had to be sure of the patient`s possibility to arrive early to our clinic in the case of a febrile episode. Otherwise, the subject had to stay in our clinic during the critical period. The patients usually stay in our hospital, because of their long distance between their residence and hospital. If the patient after the consolidation chemotherapy was discharged from the hospital, he/she had to follow these instructions:

- check their vital parameters daily (blood pressure, temperature), - avoid obviously sick people, - avoid places with large numbers of people, - dietary restriction (patient could eat only fresh and well cooked meals,) -visit our clinic weekly, - contact our clinic by phone, if any change of clinical status occurred.

If there was a change of the clinical status, patients were immediately admitted to our clinic. In most cases, it was a febrile episode, which led to the hospitalisation. Upon arrival at the clinic, a complete clinical and laboratory check was performed. In the event of 
a fever or other signs of infection, the aerobic and anaerobic blood cultures were set up as well as cultures from any presumed site of infection. After an initial evaluation, all patients were hospitalized and received broad-spectrum intravenous antibiotics in accordance with local and international recommendations until the fever subsided. After 48 hours of sustained fever, the empirical antifungal treatment was initiated. The default algorithm of the treatment of febrile neutropenia was adjusted, when necessary, according to the response, results of cultures and sensitivity tests. The patients were monitored daily for clinical complications. A complete blood cell count and biochemical tests were repeated at least 24 hours until the discharge. After several days of non-febrile period, and when clinical and laboratory signs of infection disappeared, patients were usually discharged. The same treatment and management was performed on the patients who stayed in our clinic from the beginning of consolidation chemotherapy till their discharge from hospital in case of fever. Growth factors (G-CSF) were not used routinely, but were allowed when given as a part of mobilization of stem cells before autologous stem cells transplantation.

\section{Results}

The total number of patients included in this study was 56 . There were 30 (53\%) males and 26 (47\%) females. The mean age was 46.5 years (range from 20 to 66). The number of patients, who were discharged from the hospital (outpatients) was 41 (73.2 \%), and those who stayed hospitalized (inpatients) was 15 (26.79\%). The number of patients that required the ambulatory management only (Fig. 1), e.g. platelet concentrates or red blood cells transfusions, was 17 (30.4\%). Re-hospitalization occurred in 24 cases (42.8\%). In the vast majority of cases, it was the febrile neutropenia, which led to admittance of the patient. The massive bleeding symptoms and subsequent re-hospitalization occurred in only 1 case $(1.7 \%)$. In group of inpatients, only 2 (3.6\%) courses of chemotherapy had not been complicated and the patients reached the hemopoetic recovery without a febrile episode. Among all 36 febrile episodes, the microbiologically documented infection was
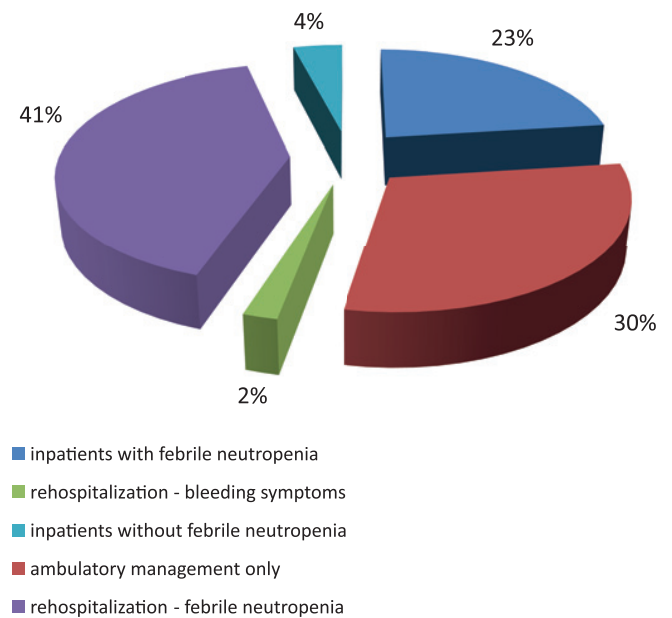

Fig. 1.Percentage of patients divided according to need and reason for hospitalization.
Tab. 1. Micro-organisms documented from the hemocultures.

\begin{tabular}{lcc}
\hline & Outpatients (41) & Inpatients (15) \\
\hline Febrile neutropenia (n) & 23 & 12 \\
\hline Micro-organism & & \\
Gram-positive (15) & - & 1 \\
Staphylococcus aureus & 1 & - \\
Staphylococcus hominis susceptible & & \\
to oxacilin & 3 & 3 \\
Coagulase-negative staphylococci & & \\
susceptible to oxacilin & 1 & 3 \\
Coagulase-negative staphylococci & & - \\
resistant to oxacilin & 2 & - \\
Streptococcus viridans & 1 & \\
Corynobacterium species & & 1 \\
\hline Gram-negative (14) & 3 & - \\
E. coli ESBL & 2 & 1 \\
E. coli & 1 & 2 \\
Pseudomonas aeruginosa resistant & 17 & 1 \\
to aminoglycosides & 2 & 1 \\
Klebsiella pneumoniae ESBL & - & \\
Enterobacter cloaceae & - & \\
Enterococcus faecalis & & \\
\hline Funghi (1) & & \\
Candida albicans & & \\
\hline Total & & \\
\hline & & \\
\hline
\end{tabular}

the most common cause of fever (61\%) and the rest of febrile episodes were of unknown origin. In 7 cases (30.4\%) of febrile outpatients, a dangerous multiresistant species in hemoculture were detected. In the group of inpatients, we confirmed 7 bacteremias caused by nosocomial species (53.8 \%). The spectrum of microorganisms documented from the hemocultures is in the Table 1.

Ten (43.5\%) outpatients treated for febrile episodes responded to the initial empiric therapy with piperacillin+tazobactam only $(n=6)$, or in combination with amikacin $(n=4)$. In the group of inpatients, only 2 (16.7 \%) patients responded to the initial antibacterial therapy with the combination piperacillin+tazobaktam and amikacin. The broad spectrum of used antibiotics and poor effectiveness of the first-line antibiotics is characteristic for this group of inpatients (Tab. 2).

Patients re-hospitalization lasted the mean 10.9 days (range 6-35). Compared to inpatients, where the mean time of hospitalisation was 30 days (range 17-38). But, the mean duration of hospitalization for inpatients from the moment they became febrile to they discharge was 14.3 days (range 7-22).

\section{Mortality}

In the group of outpatients 2 (4.8\%) deaths occurred (Fig. 2). In the first case, the patient was admitted to hospital in irreversible shock. The patient was referred to the hospital 48 hours after the onset of the fever and died within 12 hours of re-admission, despite an intensive care. This case showed that the appropriate education of the patient is crucial in the out-hospital management. In the second case, the patient developed a severe bacterial meningitis caused by ESBL Klebsiella pneumoniae, which led to his death in 48 hours after hospitalization. In the group of inpatients $1(6.6 \%)$ death due to fatal intracranial haemorrhage occurred, as the medical complication of thrombocytopenia. 
Tab. 2. Characteristics of Outpatient and Inpatient nadir episodes.

\begin{tabular}{|c|c|c|c|c|}
\hline & Total & Outpatients & Inpatients & $\mathrm{p}=$ value \\
\hline Number of patients & 56 & 41 & 15 & \\
\hline Number of febrile episodes & 36 & $23(56 \%)$ & $12(80 \%)$ & \\
\hline Male/female & & $23 / 18$ & $7 / 8$ & \\
\hline median age of years (range) & $46(20-66)$ & $44.5(20-63)$ & $50(25-66)$ & \\
\hline mean days of hospitalization (range) & & $10.9(17-38)$ & $30.6 / 14.3^{*}$ & $\mathrm{p}<0.0001 / \mathrm{p}=0.07^{* *}$ \\
\hline $\begin{array}{l}\text { Number of responses to first line ATB (Piperacillin/tazobaktam, } \\
\mathrm{P} / \mathrm{T}+\text { Amikacin) }\end{array}$ & & $10(43.5 \%)$ & $2(16.7 \%)$ & \\
\hline $\begin{array}{l}\text { Number of responses to second line of ATB } \\
\text { (Vancomycin+Ceftazidime+ Amikacin+Fluconazole) }\end{array}$ & & $6(26 \%)$ & $5(41.7 \%)$ & \\
\hline Number of responses after addition Meropenem & & $7(30.5 \%)$ & $5(41.7 \%)$ & \\
\hline $\begin{array}{l}\text { Numbers of cases when other type of antimycotics had to be added } \\
\text { to therapy (Lyposomal amphotericin B, voriconazole, posaconazole, } \\
\text { mycafungin, caspofungin) }\end{array}$ & & $6(26 \%)$ & $9(75 \%)$ & \\
\hline mortality & $3(5,4 \%)$ & $2(4.8 \%)$ & $1(6.6 \%)$ & \\
\hline
\end{tabular}

\section{Discussion}

There is little published data of the safety of the "out-hospital care“ approach in centres with limited resources. In our study, limited by a small number of patients, we wanted to contribute to already small number of relevant data. Nevertheless, our results confirm that the early discharge from hospital is feasible for those patients who meet criteria listed above. Of the 41 outpatients, 17 (41.5\%) did not require re-hospitalization at all. In contrast with inpatients, where neutropenic episodes did not occure in only 2 (13.3 \%) cases of 15 critical periods. These data (outpatients) are very similar to those, described by Naithani et al (1), despite the fact, that we did not utilise antibiotic prophylaxis and the patients had $\mathrm{n}$ adequate home care facilities. A high number of symptomatic inpatients is partly explicable by their low number.

The Table 2 shows that there was much better effectiveness of the first line antibiotics used in the outpatients group in contrast to a high consumption of broad-spectrum antibiotics and antimycotics in the inpatient group.

As the table 1 shows, multiresistant bacteria were also detected in hemoculture taken from seven outpatients in the time of their re-hospitalization. These patients probably acquired nosocomial strains during the administration of chemotherapy in hospital. For these patients, the early discharge didn't decrease the risk

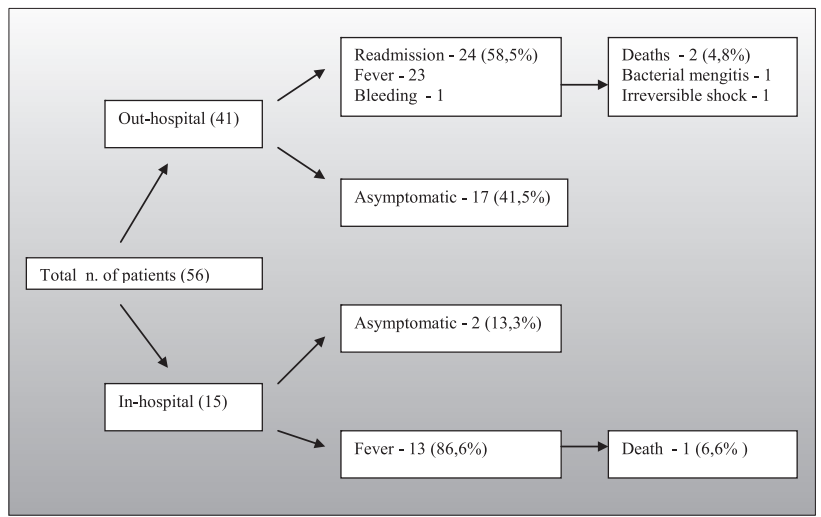

Fig. 2. The scheme shows the outcome of neutropenic episodes. of nosocomial infection. Therefore, we recommend to obtained cultivation samples before patient's discharge to ensure the most adequate antibiotic treatment in the case of readmission (3). The role of decontamination before a discharge is questionable.

We also noticed the fact that the duration of rehospitalisation did not differ from the hospitalisation of the inpatients from the time they became febrile. In other words, if we count the days from the onset of the fever to discharge, for both groups, we did not prove a statistical significance of an early discharge $(\mathrm{p}=0.07)$. This fact is probably caused by a small number of patients in the study. Despite this fact, with the out-hospital approach we saved many days of the patient's life. In conclusion, if all conditions and recommendations listed above are met, we clearly demonstrated that the early discharge is feasible, safe and cost saving option.

\section{References}

1. Naithani R, Kumar R, Mahapatra M, Agrawal N, Mishra P et al. Early discharge from hospital after consolidation chemotherapy in acute myeloid leukemia in remission: febrile neutropenic episodes and their outcome in a resource poor setting. Haematologica 2008; 93 (9): 141-168.

2. Cherif H, Johansson E, Bjőrkholm M, Kalin M. The feasibility of early hospital discharge with oral antimicrobial therapy in low risk patients with febrile neutropenia following chemotherapy for hematologic malignancies. Haematologica 2006; 91 (2): 215-222.

3. Savoie ML, Nevili TJ, Song KW, Morano SG, Ceesti F, Coppola L et al. Shifting to outpatient management of acute myeloid leukemia: a prospective experience. Ann Oncol 2006; 17: 763-768.

4. Girmenia C, Alimena G, Latagliata R, Morano SG, Celesti F et al. Out-patient management of acute myeloid leukemia after consolidation chemotherapy. Role of a hematologic emergency unit. Haematologica 1999; 84 (9): 814-819.

5. Girmenia C, Latagliata R, Tosti S, Morano SG, Celesti F et al. Outpatient management of acute promyelocatic leukaemia after consolidation chemotherapy. Leukemia 1999; 13 (4): 514-517.

6. Gillis S, Dann EJ, Rund D Selective discharge of patients with acute myeloid leukemia during chemotherapy-induced neutropenia. Am J Hematol 1996; 51 (1): 26-31.

7. Leibovici L, Paul M, Cullen M, Bucaneve G, Gafter-Gvili A et al. Antibiotic prophylaxis in neutropenic patients: new evidence, practical decisions. Cancer 2006; 107: 1743-1751. 